\title{
Neue Schweizer Arbeiten zur Glazialmorphologie des Mittellandes
}

\author{
Von Hans Graul, Heidelberg \\ Mit 2 Abbildungen im Text
}

$\mathrm{S}$ u nim a ry. In this report the most important results of 5 new Swiss papers on glacial and periglacial landforms of the "Schweizer Mittelland“ are published and discussed. These very good studies show the first geomorphological maps $(1: 25000)$ of the "Mittelland" and for the first time they analyse periglacial forms in this region. A reiterated and radical change of accumulation and erosion is rejected within the melt-water-valleys during the Würm-glaciation. The thick accumulation of pebbles is recognized as "Vorstoßschotter". Nowhere it has been noticed that fluvioglacial pebbles interfinger with end-moraines. The highest of the lower terraces is the built-uplevel of the fluvioglacial accumulation of the Würm-time. For that reason the Würm-time seems to be undissected in the Hochrhein- and Aare-valley. This opinion is opposite to J. Hug, L. ErB, D. Kimball, F. E. Zeuner and R. Huber.

The reviewer points out that real cut-terraces are only existing in relatively short parts of the valleys (C. TroLL's "Trompetentälchen“). But for the rest you can find as a rule an erosional disconformity on every" wider inset terraces-level, because there are secondary pebbles of about $5--8 \mathrm{~m}$ thickness interbedded in the older main accunulation of the Würm-time. The question whether there are floors running throughout the valley below the main terrace-level in the Hochthein valley, cannot be answered by individual and relatively small sections of a melt-watervalley; least of all in the Hochrhein valley above the mouth of the Aare. For the development of deeper situated lower-terraces was controlled by the later advance of the Rhone-glacier at a time, when the other Swiss glaciers were receding. The reviewer substantially agrees with the Swiss scheme of periglacial forms and the classification of the Riss-time.

In den letzten Jahren hat die glazialgeologische und -morphologische Bearbeitung des Schweizer Mittellandes eine beachtliche Belebung erfahren, die auf die Anregungen von H. Annaheim-Basel (auch als Vorsitzendem der rührigen Schweizer Geomorphologischen Gesellschaft) und H. Bossch-Zürich zurückgeht. Die hervorragenden Schweizer Untersuchungen der 90 -er bis 30-er Jahre hatten vielleicht gerade wegen der wissenschaftlichen Autorität mancher ihrer Bearbeiter eine gewisse Hemmung für die weitere Entwicklung der Schweizer Quartärforschung bedeutet, zum anderen fehlte ihr bis zu einem gewissen Grade die Untersuchungsmethodik des Geomorphologen, wie sie in Deutschland durch die Schulen von A. PENCK und E. BRüCKNEP schon seit Beginn dieses Jahrhunderts eine ganz besondere Pflege erfahren hat. Und wenn auch die deutsche Periglazialforschung von den Geologen W. SAlomon, W. Soergel und P. Kessler ausgegangen war, so fand sie doch frühzeitig an einer ganzen Reihe von Geographischen Hochschulinstituten eine Heimstätte höchst intensiver Pflege. In der Schweiz aber war dieser Zweig der Quartärforschung bis zum Ende des 2. Krieges fast unbekannt geblieben.

Du Pasquier, F. Mühlberg, J. Hug, Alb. Heim, R. Frei, P. Beck und viele andere Schweizer Quartärforscher waren Geologen gewesen. Die hier zu besprechenden Arbeiten stammen aber durchweg von jungen Schweizer Geographen. Ich würde dieses Faktum nicht so herausstellen, wenn damit nicht verbunden wäre die Tatsache einer auffallend neuen Perspektive in so manchen Fragen des Mittelland-Quartärs. Die neuen Untersuchungsmethoden und Ergebnisse stehen denen, welche in den letzten Jahrzehnten im schwäbisch-bayrischen und österreichischen Alpenvorland erzielt worden sind, weit näher als manche der früheren Auffassungen. Dies ist vor allem auch der Grund für mich, hier auf jene neuen Schweizer Arbeiten besonders hinzuweisen, wobei nicht bloß an eine Berichterstattung der genannten Arbeiten, sondern an eine auch kritische Betrachtung derselben gedacht wird. Es soll hier also über die neuen Ergebnisse diskutiert werden, was ich glaube mir erlauben zu dürfen, da ich mich in den letzten Jahren ziemlich intensiv mit dem Jungpleistozän des Mittellandes beschäftigt habe. 
Es handelt sich um folgende Veröffentlichungen:

1) JäCKLI, H.: Talgeschichtliche Probleme im aargauischen Reußtal. - Geographica Helvetica 11, S. 46-59, 1 morpholog. Karte 1 : 50 000, 3 Abb., Zürich 1956.

2) LeEmann, A.: Revision der Würmterrassen im Rheintal zwischen Diessenhofen und Koblenz. - Geogr. Helv. 12, S. 89-173, 23 Abb., Zürich 1958.

3) Bugmann, E.: Eiszeitformen im nordöstlichen Aargau. - Mitt. d. Aargauischen naturforsch. Gesellsch. 25, 94 S., morpholog. Karte 1:25000 in zwei Blättern, 13 Abb., Aarau 1958.

4) Moser, S.: Studien zur Geomorphologie des zentralen Aargaus. - Mitt. d. geographisch-ethnologischen Gesellsch. Basel 10, 98 S., morpholog. Karte 1 : 25 000, 5 Beilagen, 9 Photos, 18 Abb. im Text, Basel 1958.

5) Annaheim, H., Bögli A. \& Moser, S.: Die Phasengliederung der Eisrandlagen des würmeiszeitlichen Reußgletschers im zentralen schweizerischen Mittelland. - Geogr. Helv. 13, S. 217-231, Zürich 1958.

Etwa zu gleicher Zeit sind auch mehrere Arbeiten zum gleichen Thema von geologischer Seite vorgelegt worden. Ich nenne vor allem R. HubER (1956), H. BECK (1957) und R. Hantke (1959 a und b). Sie weisen aber außer Hantke's Arbeiten zu wenig Kontakt mit den hier zu besprechenden Dingen auf und sollen daher in einem anderen Rahmen des näheren behandelt werden. Im Übrigen ist die Arbeit von R. Huber bereits einer kritischen Betrachtung bei A. LeEmann unterzogen worden. Diese Veröffentlichungen wie eine Diskussion zu 5) (H. TäcKLI 1959) wie alle im folgenden zitierten Arbeiten sind im Literaturverzeichnis aufgeführt.

In den fünf genannten Untersuchungen werden eine ganze Reihe neuer Ergebnisse erzielt, von denen folgende besonders herausgestellt sein mögen:

1. Zum ersten Male werden nach einer Geländeaufnahme bis zu $1: 5000$ herab morphologische Karten auf der topographischen Grundlage der neuen Schweizer Landeskarte $1: 25000$ eines größeren Ausschnittes des Mittellandes vorgelegt. Es handelt sich um das Gebiet von der Aaremündung bis kurz vor Aarau, nach SE bis zur Lägern, südl. Bremgarten, Dottikon und Seon.

2. Zum ersten Male wird aus dem Schweizer Mittelland eine reichhaltige Serie von periglazialen Formen der letzten Eiszeit beschrieben und kartiert (vor allem LeEmanN, Bugmann und Moser). Es werden die Formen auf letzteiszeitlichen Ablagerungen analysiert und versucht, sie als hoch-, soät- oder postglazial zu erkennen (L. und B.). Zu den ersteren gehören alle jene, deren Entstehung auf Bodenfließen zurückgeführt werden kann, so "Hangdellen“ (L.), die M. als "muldenartige Einbuchtungen in der Terrassenkante“ bezeichnet, deren Ausraum als deutlicher Schuttkegel auf der nächsttieferen Terrasse abgelagert wurde. Auch B. beschreibt diese Formen von der Leuggerner Terrasse (linke Seite, unteres Aaretal) als "Kantenkerben" oder "Miniaturdellen". Auch diese streichen in die Luft aus (nach seiner Fig. 16). L. sieht darin einen Beweis, daß die darunter gelegene Terrassenfläche (z. B. Hasli-Terrasse bei Kaiserstuhl im Rheintal) eine jüngere Erosionsfläche in der Würm-Akkumulation ist.

Ferner werden „asymmetrische Muldentälchen“. (L.) oder „Dellen“ (M.) oder „Hangdellen" (B.) beschrieben, die weiter hangwärts reichen. Im Altmoränengebiet sollen Dellen auch durch mächtige Auffüllung aus Kerbtalformen entstanden sein (B.). Eine dritte noch hochglaziale Form stellen die Korrasionstäler mit kastenförmigem Querschnitt dar (L.). Hingegen schon spätglazial, nach entsprechend tieferem Auftauen des Dauerfrostbodens, sollen alle Hohlformen mit V-förmigem Querschnitt sein, also mit fluviatiler Formung, so "Gräben“ (L.), und „größere Kerben“ mit Schwemmkegel auf tieferer Erosionsterrasse (B.). Diese Formen sind heute in der Regel trocken. Die noch durchflossenen zeigen größeres Einzugsgebiet, tiefere Einschneidung, also direkten Kontakt mit der rezenten Ero- 
sionsbasis und sind holozän. Nur M. hält auch „fluviatile Miniaturtälchen“ für noch hochglazial, da er solche auf seinem noch hochglazialen C-Talboden des Aaretals (bei Rupperswil) auslaufend beobachten konnte. Eine Kiesgrube am Hang dieses Tälchens zeigt wahrscheinlich Taschenböden (S. 49).

4. Der an sich bekannte Formunterschied zwischen mittel- und jungpleistozänen Terrassenflächen wird einer erweiterten Betrachtung unterzogen, welche die besonderen Schweizer Verhältnisse berücksichtigt. Diese sind gegeben durch die Uberfahrung und Uberarbeitung der meisten mittelpleistozänen Vorstoßschotter durch das Eis mindestens der Maixmalvergletscherung (=Riß II der Schweizer).

5. Von Bugmann wird die richtige Genese des Surbtales erkannt, das während des letzten Glazials zuerst von einer mächtigen Lokalschüttung aufgefüllt wurde, der sich erst bei Überfahrung der Wasserscheide (zur Glatt bei Schöfflisdorf-Sünikon) durch den Gletscher das fluvioglaziale Fernmaterial deutlich beimengt. Es ist dies eine schöne Parallele zu dem Fazieswechsel der Schotter im oberen Donautal beim Vorstoß des rißzeitlichen Rheingletschers über die Rhein-Donau-Wasserscheide (Graul 1952, 1953). Auch wird von B. richtig erkannt, daß es sich bei der Akkumulation in den autochthonen Tälern um eine selbständige Aufschotterung des Glazials handelt (dieser weiße Jurakalkschutt wird hier "Chäppeligrien“ genannt), also um eine klimatisch bedingte, periglazial-fluviatile Akkumulation, wie sie bei uns seit den Untersuchungen von W. SoERGEL (1920) bekannt ist.

6. Mit einer „Revision der Würmterrassen“ (LeEmanN) wird das Hug-Erb-KIMbalLZEUNER-HUBER'sche Schema von mehreren ineinandergeschachtelten Niederterrassen-Akkumulationen im Hochrhein- und im unteren Aaretal abgelehnt (B. und M.). Es wird nach der im Geographischen Institut der Universität Zürich ausgearbeiteten "Indizmethode“ (vgl. H. BoEsch 1957) der Nachweis versucht, daß es nur e in e Würm-Akkumulation in diesen Tälern wie in deren Nebentälern gegeben habe mit der Akkumulations-Endfläche als oberste Niederterrasse, während alle tiefer gelegenen Flächen reine Erosionsflächen seien.

7. Für diese letzteren wird mehr oder weniger eindeutig abgelehnt, daß sie sich als Reste einheitlicher Talböden durch das ganze Hochrheintal wie auch durch das Aaretal verfolgen lassen. Es handle sich vielmehr vielfach um „abgleitende Erosionsflächen“, ähnlich wie Gleithänge bei Gleitmäandern (so vor allem LEEMANN). Moser findet dagegen eine "präzis von den übrigen Flächen zu trennende Terrasse“ im Aaretal, etwa 15-20 m unter dem Würm-Akkumulationsniveau.

8. Die extramoränen Schotter unter diesem obersten Niederterrassenniveau werden als einheitliche Ablagerung der letzten Eiszeit erkannt, nämlich als Vorstoßschotter (L., B. und M.). Eine Unterlagerung dieser Vorstoßschotter durch einen älteren „Mittelterassenschotter", wie dies noch JäcKLI für das untere Reußtal, R. Huber (1956) für das Rafzerfeld und im unteren Glattal annehmen, wird für das gesamte Untersuchungsgebiet abgelehnt, da in keinem Aufschluß dafür ein entsprechender geologischer Beweis erbracht werden könne.

9. Die Würm-Vorstoßschotter verzahnen sich nirgends mit Endmoränen, sondern sie werden von diesen auf unruhiger Erosionsfläche überlagert (M. und L.). Moser legt ein neues Blockbild für die Penck'sche "Glaziale Serie“ vor, welches seinen Beobachtungen Rechnung trägt.

10. Die von A. Bögli schon 1954 vorgeführten überfahrenen Jungmoränen südlich Seon werden von Moser bestätigt. Auch im Bünz- und im Reußtal werden von ihm solche überfahrenen Jungmoränen erkannt und kartiert. Sie haben nichts mit den von J. KNAUER (1954) beschriebenen überfahrenen Moränen von Zürich und Bremgarten zu tun, die im Gegenteil als Rückzugshalt aufgefaßt werden wie bisher. Dieser Auffassung schließt sich auch R. Hantke (1959) an. Nach Moser entsprechen die Zungenbecken „keineswegs den 
schulmäßigen Vorstellungen eines mit Grundmoränen ausgekleideten und mit Drumlins übersäten Beckens". Die Grundmoränen und drumlinoiden Formen werden eben vielmehr als überfahrene Jungmoränen aufgefaßt.

11. Während BugmanN und LEemanN im extramoränen Bereich nicht einmal Beweise für eine Phasengliederung der letzten Eiszeit finden, sieht Moser in der Gruppierung der Moränenwälle und der von ihnen ausgehenden verbreiteteren Erosionsflächen den Nachweis für Schwankungen im Hochglazial, von denen in seinem Untersuchungsgebiet drei Eisvorstöße mit längerem Halt zu erkennen sind:

F -Moränen (überfahrene), so z. B. Seengen,

M-Möränen (die äußerste Gruppe), so z. B. Seon,

$S$-Moränen (innerhalb der F-Gruppe), so z. B. Hitzkirch.

Meine bisherigen Beobachtungen bestätigen diese Gliederung weitgehend.

12. Mit dem Ende des Gletscheranwachsens zum äußersten Stand setzte am Gletscherende die Tiefenerosion ein und zerschnitt Moränen und das oberste Schotterfeld mit Trompetentälchen im Sinne C. TROLL's (1926). Diese Auffassung ist bei uns seit der präzisen Darstellung C. TroLL's eigentlich allgemein gültig.

13. Über den Wechsel von Erosion und Akkumulation in der glazialen Schmelzwasserrinne während eines eiszeitlichen Klimazyklus' ist man sich nicht völlig einig. Während Moser sich an die Auffassungen von I. SChaffer (1950) anlehnt, lehnen Bugmann und LeEmann sie für ihre Gebiete ab, wobei letzterer ein sehr interessantes Querprofil des Rafzer Feldes vorlegt, das die ablehnende Haltung zu begründen vermag.

14. Es werden - wie bisher in der Schweiz - zwei große Rißvorstöße des Eises angenommen, von denen der zweite der maximale war. Die Verhältnisse liegen also gleich denen im nordwestlichen Rheingletschergebiet. Zwischen beiden Vorlandvereisungen soll aber nach Moser kein ausgesprochenes Interglazial geherrscht haben, womit er eine Mittelstellung zwischen den Auffassungen von R. FrEI einerseits und Fr. MüHLBERG andererseits einnimmt.

15. Aus dem Betrag der Tiefenerosion in den Schmelzwasserrinnen kann nicht die Dauer eines Interglazials berechnet werden (Moser nach SCHAEfer). Doch sind die Schottersohlen-Differenzen zwischen Jüngerem Deckenschotter und Hochterrassenschotter in den auf S. 87 bei Moser angegebenen Stellen um 40-80\% größer als zwischen Alterem Dekkenschotter und Jüngerem. Es kommen hier die besonderen tektonischen Vorgänge im Schweizer Mittelland zur Wirkung (vgl. schon E. Brückner). Außerdem wird - für das Mittelland ebenfalls ein selten geäußerter Gedanke - auf die Bedeutung der glazialen Erosion im Mittelland während der mittelpleistozänen Vereisungen wenigstens hingewiesen (Moser, S. 81).

16. Ein besonderes Problem stellt die Datierung des nicht mehr mit quartären Ablagerungen bedeckten Flachreliefs dar. Moser kartiert System-Talkanten und Strukturformen wie „Schichtrippenkanten“ und „Härtekanten“. Erstere gliedert er nur in präglaziale und glaziale, wie er auch beim Flachrelief nur präglaziale Flächen (z. B. den Bözberg) ausscheidet. Bugmann hingegen datiert einen großen Teil auch des jüngeren Flachreliefs und zwar in der Weise, daß er die von Quartärablagerungen denudierte Fläche nach ihrer Höhenlage mit der nächsthöheren Schotteroberfläche zeitlich korreliert. Im Text äußert Moser eine ähnliche Auffassung (S. 70).

17. Es werden noch eine Reihe flußgeschichtlicher Tatsachen, Epignesen, Anzapfungen etc. behandelt, auf die hier aber nicht näher eingegangen werden soll.

$\mathrm{Zu}$ einzelnen Punkten dieser Ergebnisse möchte ich mir folgende Bemerkungen erlauben:

$\mathrm{Zu}$ 2. Es wird vorgeschlagen, für die Formen der periglazialen Denudation möglichst eine einheitliche Terminologie zu verwenden. Dellen sind denudative, Täler erosive Formen; von „nichtfluviatilen Miniaturtälchen“ zu sprechen ist aber eine Kontradiktion. 
Die Tiefe des in die Terrassenfläche eingelassenen Dellenbodens sagt nichts über die Tiefe des Auftaubodens aus. Während der Dellenbildung ging nämlich die Tieferlegung des Dellenbodens parallel mit der des sommerlichen Auftaubodens. Bei etwa gleichbleibender Tiefe des Auftaubodens kann sich also die Delle dauernd vertiefen und verbreitern.

Wichtig ist die Beobachtung von Moser, daß die Vorform der Hangdellen kleine muldenförmige Einbuchtungen in der Terrassenkante sein dürften. Es erscheint naheliegend anzunehmen, daß alle diese Formen an gewisse Konzentrationen des Auftauwasseraustritts an der Terrassenkante gebunden sind. Das Auftauwasser erfüllte den gesamten Auftauboden, bewegte diesen aber nicht gleichmäßig in der Fallrichtung der Terrassenoberfläche, sondern in bestimmten Linien oder Streifen stärker, so daß es an der Terrassenkante nicht zu einem gleichmäßigen geschlossenen Abfließen der Fließerde kam, sondern zu einem in kleinen Hangdellen mit entsprechendem Schuttvorbau auf dem Terrassenhang. Die Tiefe, Größe und Länge der Dellen ist weniger eine Funktion der Zeit, als vielmehr die Funktion der Menge des zufließenden Wassers in diese Dellen, eine Funktion, die der Selbstverstärkung unterliegt. Diese zufließende Wassermenge war am größten unterhalb von Hohlformsystemen im Rückhang der Niederterrassenfläche (siehe dazu Fig. 16 bei BuGMANN). Je nach Zusammensetzung des Schotters und der Mächtigkeit seiner Fließerdebedeckung (auf die Tonsubstanz des Auftaubodens kam es also an) konnten die Korrasionsströme in den Dellen länger oder kürzer der formgebende Faktor sein. War der Wasserzufluß aber stärker, dann konnte es bei einem bestimmten Grenzwert auch zur Bildung eines oberflächlichen Gerinnes und zur Erosion kommen, wie sie Moser im N des Schafisheimer Feldes als noch hochglazial ansprechen möchte. Tatsächlich ist die tiefere Rupperswiler Terrasse (Langi Tanne und Hard) noch hochglazial, wie ich auf anderem Wege nachweisen kann. Allerdings zweifle ich bei den von Moser geschilderten Formen, daß es sich um solche von richtigen Gerinnen handelt. Es hat in der Kaltzeit alle Übergänge von langsam fließendem bis ruckartig schnell abfließendem Auftau-Schuttbrei in den Dellen gegeben, die nach dem Wasser-Schutt-Verhältnis die Reihe von der Delle über asymmetrische kastenförmige Korrasionstäler bis zu symmetrischen kastenförmigen Tälern entwickelten, von denen die letzten Formen dieser Reihe vorwiegend fluviatil geformt wurden (vgl. dazu schon die eingehenden Betrachtungen von J. BüDEL 1944).

Zu 3. Die Tatsache, daß ein Dellenboden im Hanggelände über den ihm vorgelagerten Terrassenboden in die Luft ausstreicht, entscheidet für sich allein noch nicht, ob die vorgelagerte Terrassenfläche jünger ist als die Entstehung der Delle (LeEmann). Nur wenn der zu der Dellenbildung gehörige Schuttkegel am Ausgang der Delle fehlt, was nur möglich ist, wenn der Schuttkegel durch das Schmelzwasser des Haupttales weggeräumt wurde, dann ist der vorgelagerte Terrassenboden jüngerer Entstehung als die Delle. Ob dieser Terrassenboden aber eine erosive oder eine akkumulative Fläche ist, wird damit allein auch noch nicht entschieden. So sind jedenfalls die von Leemann beschriebenen Dellen über der Hasli-Fläche und die Korrasionstäler am W-Ende des Rafzerfeldes älterer Entstehung als die von Bugmann beschriebenen Formen auf der Leuggerner Terrasse, obwohl beide Formen auf die gleiche Ursache, nämlich auf Denudation durch abfließenden Auftauboden zurückgeführt werden. Man erkennt daraus abermals, daß selbst diese kleinen Formen vielseitige Ursachen haben, also nicht allein das Vorhandensein eines Dauerfrostbodens, und daß mit ihrer Hilfe eine allzufeine Datierung nicht möglich ist.

Eine Reihe von Beobachtungen (vor allem von Bugmann und Moser) weist darauf hin, daß der Dauerfrostboden und damit die hochglazialen Klimaverhältnisse im Schweizer Mittelland noch nach Beginn des Einschneidens von Aare und Hochrhein in ihr oberstes Aufschüttungsniveau wirksam gewesen sein müssen, was zu erwarten war.

Zu 5. Auch die Beobachtung, daß die Schwemmkegel des Chrüzlibaches bei Reckingen auf eine tiefe Rhein-Niederterrasse ausmünden, (es ist die Zurzacher, welche nach L. ERB 1936 und Kimball \& ZeUner 1946 der unteren NT entsprechen und altersgleich mit der 
Inneren Jungmoräne sein soll) beweist, daß die kalt-klimatische Akkumulation der autochthonen Vorlandflüsse in der Regel länger währte als die der fluvioglazialen Schmelzwässer (Bugmann). Es erscheint daher sicherer, die Dauer des Hochglazials nach der Andauer der Akkumulation in den periglaziären Tälern zu bestimmen als nach der Andauer der Bildung der Glazialen Serie irgendeines Gletschers (ähnlich schon Soengel).

Zu 6. Zu dem Punkt „Revision der Würmterrassen“ (Leemann) wäre viel zu sagen. Jedenfalls stellt das Ergebnis, daß weder im Hochrhein- noch im Aaretal mehrere ineinandergeschachtelte mächtige Schotterablagerungen der Würmzeit bestehen, einen großen Fortschritt dar, der m. E. auf die alten Auffassungen von Du PAsQuier, A. PENCK und E. BRüCKNER zurückführt. Daß man sich gegen allzu kühne Korrelationen von Terrassenflächen über größere Lücken $(8-10 \mathrm{~km})$ hinweg oder gegen eine "Höhenzahlenakrobatik" (BugmanN) wendet, ist durchaus zu unterstuitzen. Aber es sollte doch immer etwas bedenklich stimmen, wenn man bei seinen Beobachtungen und Schlüssen zu einer ausgesprochenen Antithese gegenüber den bisherigen, oft seit mehreren Jahrzehnten gültigen Thesen gelangt. Meistens ist doch nicht alles falsch, was bisher gesehen und gedacht wurde. So sprechen weder A. Penck noch E. Brückner, noch C. Troll oder im Spezialfall auch nicht L. ERB im Hochrheintal von mehreren würmzeitlichen Akkumulationen nach Ausräumungsphasen jeweils bis zum Präwürmtalboden herab. Dies meinten vielleicht J. Hug (1909), bestimmt Kimball \& Zeuner (1946), deren wichtige Arbeit aber garnicht bekannt gewesen zu sein scheint. Die sehr eingehenden Überlegungen (vor allem bei Lfemann) zu diesem Problem sind sehr zu begrüßen; aber ich hätte doch folgendes zu bemerken:

a) Der Talabschnitt Aaremündung-Schaffhausen ist der ungünstigste im ganzen Hochrheintal, um das Problem seiner Niederterrassen-Gliederung zu lösen.

b) Diese Frage kann nicht von einem Einzelabschnitt aus beantwortet werden, auch wenn man noch so wichtige Gründe gegen großräumige Untersuchungen besitzt. So groß ist das Gebiet übrigens nicht, daß nicht mit den gleichen minutiösen Methoden, wie sie in den kleineren Abschnitten angewandt worden sind, erfolgreich gearbeitet werden könnte, notfalls mit einem Teamwork.

c) Auch im Aaretal ist diese Frage nicht vom unteren Talabschnitt aus zu lösen; oberhalb der Wildegger Enge wird die Sache nämlich erst richtig interessant.

d) Es gibt e in e n bestimmten Abschnitt in jeder Schmelzwasserrinne, in dem viele Terrassenflächen talab auf eine oder auch mehrere Flächensysteme k o n v e r g i e r e n. Es ist der Abschnitt unmittelbar im Endmoränenbereich und eine Strecke unterhalb davon: der Abschnitt der Teilf elder (A. PENCK), der Ausgleichstiefenerosion beim Zurückweichen des unteren Gletscherendes, der Trom pe ten tä $1 \mathrm{ch}$ en (C. Troll). Die Teilfelder sind in ihren oberen Teilen ohne Zweifel reine Erosivformen (C. TRoLL). Aber neben dieser Erosion im glazialen Oberlauf der Schmelzwasserrinnen (I. SCHAEfer) setzt selbstverständlich überall dort, wo die Geröllmassen aus jener Einschneidungsstrecke und die der weiterhin akkumulierenden Nebenflüsse das Belastungsverhältnis des Hauptflusses nicht überlasten, Erosion ein: nämlich die Erosion von der un teren Erosionsb a s is aus.

Zwischen ihr und der oberen Erosionsstrecke können an verschiedenen Stellen, insbesondere am Austritt von Engtalstrecken, auch örtliche Erosionsbasen wirksam werden. Es entstehen allenthalben neue Einschnitte in die Akkumulations-Endfläche und Erosionsflächen. Die wenig gestörte oberste Akkumulationsfläche wird nun abgelöst durch eine mehrfach gestufte Gefällslinie, die nicht überall flacher zu sein braucht als jene. Im Gegenteil treten an den Stellen einsetzender Tiefenerosion steilere Talweggefälle auf, als vorher bestanden. Diese Entwicklung etwa meint Leemann im Hochrheintal oberhalb der Aare-Einmündung erkennen zu können. Er kann die verschiedenen Talböden seiner zwei 
gesondert behandelten Unterabschnitte nicht oder nur eigentlich mit $\mathrm{Zwang}$ korrelieren. Aber andererseits ist es gewiß nicht einfach so, daß bisher "größere, nicht in das System passende Formen ... als Teilfelder bezeichnet, andere für die Gliederung ganz vernachlässigt “ werden (Leemann, S. 167). A. Penck, J. Hübscher, J. Meister u. a. und zuletzt R. Huber haben ihre Terrassengliederungen im Hochrheintal in Verbindung mit den Eisständen und Moränenwällen, also von hier talab, durchgeführt. J. Hug, F. ZINK u. a. begannen mit der Gliederung an der unteren Erosionsbasis im Oberrheingraben. Es mußte beim Versuch einer Verknüpfung der beiden Untersuchungswege (so vor allem durch L. ERB und Kimball \& ZeUNer) zu gewissen Schwierigkeiten in jenem Zwischenstück kommen, wo die Wirkungen der unteren Erosionsbasis und die Erscheinungen der Teilfelderbildung einander begegneten. Daher auch die Korrekturen von ERB an Hug, aber auch LEEMANN mußte jenen auf einige fehlerhafte Verknüpfungen aufmerksam machen (und ich könnte jetzt diese Korrekturen auch an LeEmann fortsetzen). Zu der Tatsache, $\mathrm{daß}$ sich gerade oberhalb der Aaremündung zwei vollkommen verschiedene Vorgänge nach Beendigung der Hauptakkumulation begegnen, kommen noch morphologische $\mathrm{Be}$ sonderheiten des Hochrheintales hinzu, die bei den Schmelzwasserrinnen des deutschen Alpenvorlandes fehlen. Es handelt sich um den gesteins- und tektonikbestimmten Wechsel von Engen und Weitungen, um die Veränderungen, welche durch die Überfahrung eines großen Teils des Hochrheintales durch das Eis der Maximalvergletscherung verursacht worden waren, wie schließlich um eine ziemliche Anzahl quartärer Epigenesen. So beobachten wir am Ausgang jeder bedeutenderen Engtalstrecke Teilungen bestimmter Talböden. Gerade diese Erscheinung aber ist für den Morphologen, der von einer Talweitung zur nächsten eine Terrassenkorrelierung versucht, eine häufige Ursache von Irrtümern, solange er die Genese derselben nicht voll erkannt hat. Selbstverständlich ist das obere Niveau einer solchen "Talbodenspaltung “ das ältere, und zwar ein Akkumulationsniveau. Das tiefere ist während des Abklingens der Aufschüttung gebildet worden, als die lokale Erosionsbasis der unterhalb gelegenen Weitung bereits zur Wirkung gekommen war, aber nicht durch die Engtalstrecke hindurch bis zur nächsten Weitung, an deren unterem Ende (=Engtaleingang während der gleichen Zeit) noch immer Stauakkumulation bestand, zur Wirkung gekommen war. Es besteht also an allen ähnlichen Stellen eine Art Teilfelderbildung, deren Ursache aber in dem Wirksamwerden einer lokalen Erosionsbasis bestand. Siehe dazu als Beispiel Abb. 1, einen Ausschnitt aus dem Hochrhein-Tallängsprofil mit N.T.-Flächen verschiedener Genese.

e) Es ist also vollkommen richtig, wenn LeEmann sagt (S. 170): „Die Differenzierung von Breiten- und Tiefenerosion ist nicht durchwegs an klimatische Faktoren gebunden; lokale Erosionsbasen können auch im Spätglazial eine breitflächige Erosion bedingen.“ Nur muß man bei allen diesen fluviatilen Vorgängen die Aufschüttung nicht außerhalb lassen, und zweitens fallen alle diese Vorgänge noch ins Hochglazial. Der Beweis für letztere Behauptung, die ich demnächst in einer längeren Darstellung des näheren begründen werde, kann im Aaretal erbracht werden. Moser spricht im Gegensatz zu LeemanN's großer Korrelierungsskepsis davon, daß sich im mittleren Aaaretal „eine präzis von den übrigen Flächen zu trennende Terrasse dehnt". Wer diesen Talboden aaretal-aufwärts verfolgt, wird feststellen, daß er sich oberhalb Olten in den Trockentalboden von Wangen-Bipp fortsetzt und damit bis an und unter die Jungendmoränen des östlichen Rhonegletschers. Er gehört also zur Glazialen Serie desselben, ist hier also hochglaziale Aufschüttungsfläche. Und die Fortsetzung talab soll eine Erosionsterrasse sein, bzw. sich nicht ins Hochrheintal fortsetzen?

Nein, diese wie andere über weite Strecken erhaltene Terrassenniveaus mit einheitlichem $\mathrm{fl} \mathrm{a} \mathrm{ch} \mathrm{e} \mathrm{m} \mathrm{Gefälle} \mathrm{in} \mathrm{tieferer} \mathrm{Position} \mathrm{als} \mathrm{die} \mathrm{oberste} \mathrm{Niederterrasse} \mathrm{können} \mathrm{nicht}$ nur "abgleitende Erosionsflächen“ (LEEMANN) sein. Allerdings treten sie im Hochrheintal erst unterhalb der Aaremündung übersichtlicher auf, da eben die Ursachen ihrer Entste- 


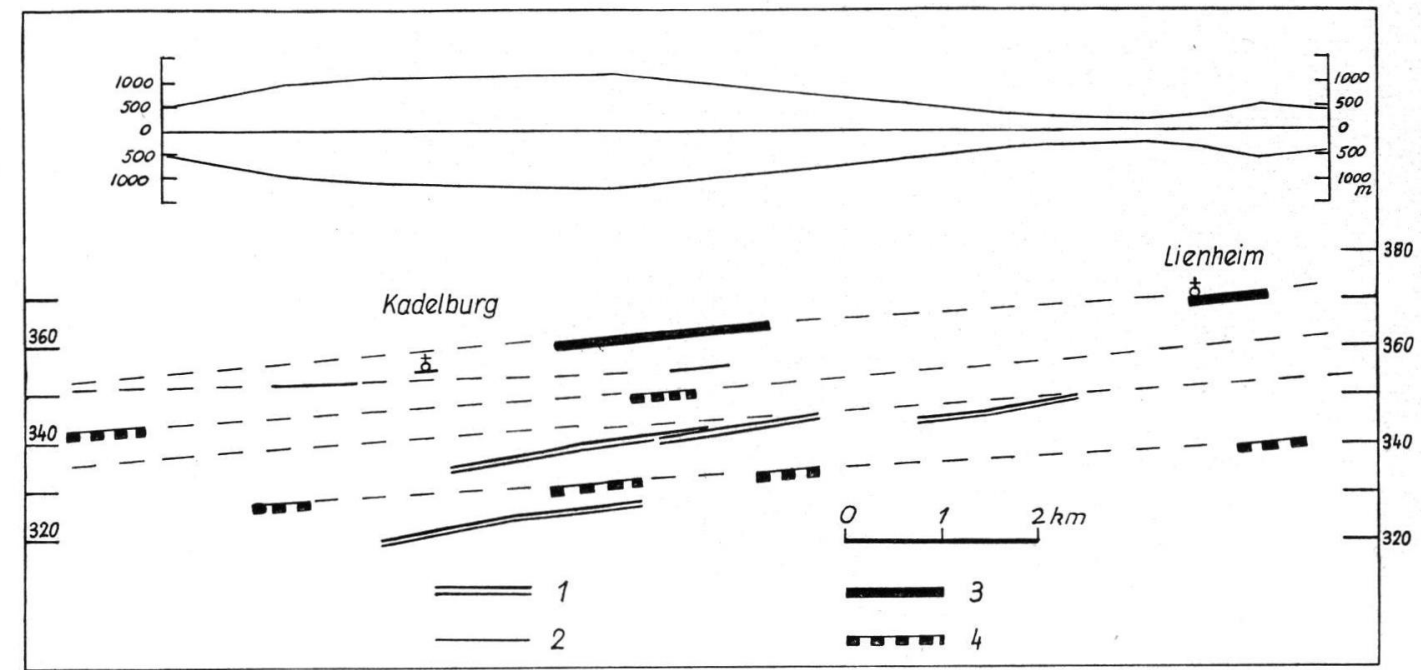

Abb. 1. Die Oberflächen der Niederterrassen im Hochrheintal zwischen Zurzach und Mellikon. Oben ist die Breite des Niederterrassen-Talbodens angegeben. 1) $\mathrm{Zu}$ steil abdachende Flächen; 2) Teilfelder; 3) Hauptakkumulationsniveau; 4) tiefere Akkumulationsniveaus. Die zu steil abdachenden Flächen innerhalb des Niederterrassensystems können jeweils nur mit ihren oberen Enden mit flachen Talbodenresten zu einem durchlaufenden Talboden verbunden werden. Bei einer Reihe von zu steilen Terrassenflächen (wie zwischen Zurzach und Mellikon) ist die talauf gelegene jünger als die talab gelegenen. Es handelt sich um eine Art Teilfeldersystem, das aber an seinem unteren Ende nicht auf einen Akkumulationstalboden mündet (also konvergiert), sondern auf eine sich in ihrer Höhenlage verschiebende, meist sich vertiefende Erosionsbasis. Es kommt also in diesem Falle nicht immer talab zu einer tatsächlichen Konvergenz der Teilterrassenfiächen.

hung nicht im Bereich der Rhein- und der Glatt-, Linth- und Reußgletscherzungen, sondern des Rhonegletschers lagen.

f) $\mathrm{Daß}$ der Charakter der Würm-Akkumulationsfläche im Sinne der Schweizer Verfasser doch nicht immer ganz einwandfrei zu erkennen ist, bestätigen einige höchstwahrscheinlich falsche Kartierungen derselben. Diese liegen meist an den Rändern der Karten. Es handelt sich um folgende Flächen: bei Bugmann die Herdlenfläche bei Leuggern, die an ihrem höchsten Punkt etwa $6 \mathrm{~m}$ unter dem obersten NT-Niveau liegt und viel zu steil abfällt. Auch die „Hard“ südlich Leuggern liegt tiefer, ebenso die Eiemer Hard (ca. $7 \mathrm{~m}$ zu tief). Diese Fläche gehört vielmehr zum System der oberen Stilli-Terrasse (Niderhard auf der linken Aaretalseite). Bei Moser: Die schmalen Terrassen von Schinznachdorf bis südlich Veltheim auf der linken Aaretalseite sind ebenfalls tiefergeschaltet und stellen nach ihrem zu steilen Gefälle nicht mehr den obersten Würm-Aufschüttungsboden dar. Auch LeEmann stellt eine zu tiefe Fläche, nämlich die von Tüftel-Rütenen bei Koblenz, ins Hauptniveau, obwohl sie $8 \mathrm{~m}$ unter diesem liegt. Das Hauptniveau setzt sich unterhalb der Aaremündung in die gut erhaltene Bernauer Terrasse fort, so daß sie bei weitem kein so großes Gefälle hat, wie L. meint.

g) Selbstverständlich ist die Indizmethode richtig. Sie ist eine geologisch-stratigraphische Methode, mit der diskordante Lagerung verschiedener, aber sich petrographisch sehr ähnelnder Straten (Fluvioglazialschotter) erkannt werden kann. Ich verwende eine ähnliche Methode seit langem, aber immer in Verbindung mit der quantitativen petrographischen Geröllanalyse (1952, 1953). Es ist nicht immer einfach, die Diskordanzen in den Schottern zu erkennen, da fossile Bodenbildungen der Liegendschotter nie (!) zu finden sind. Fluviatile Überlagerungen sind nämlich stets mit kräftigerem Abtrag der Oberfläche des Liegenden verbunden als Gletscherüberfahrungen. Diese können, aber nur 
in der Nähe des Gletscherendes, tatsächlich so behutsam über das Liegende hinweggleiten, daß dessen Bodenprofil wenigstens teilweise unter den Moränen erhalten bleiben kann (viele Beispiele aus dem deutschen Alpenvorland bekannt, so vor allem bei H. Graul, I. Schaefer \& F. Weidenbach 1951).

Die meisten tieferen Niederterrassenflächen zeigen im Gegensatz zum obersten WürmTalboden, und zwar in beliebiger Entfernung von den unteren Gletscherenden, eine mit Grobgeröll einsetzende diskordante Einlagerung, die zum äußeren Talbodenrand auskeilt. Dieses Auskeilen erfolgt in der Regel wenige bis einige Zehner von Metern vor der äußeren Kante zum nächsthöheren Terrassenhang. Die Seitenerosion, welche diesen Talbord schuf, umfaßte also oft einen etwas breiteren Raum als die neuerliche Aufschüttung. Diese wird bis zur Talmitte $5-8 \mathrm{~m}$ mächtig, beträgt aber immerhin doch noch etwa $1 / 5-1 / 6$ der Hauptakkumulation im gleichen Talquerschnitt (siehe Abb. 2). Das Material zeigt eine ausgelesenere, mehr Ortliches enthaltende Mischung, ist weniger sauber gewaschen, enthält also mehr Flußtrübe als Zwischenmittel. Gerade in Aufschlüssen, die von LeEmann und BugmanN als Beweis für den reinen Erosionscharakter der tieferen Terrassenflächen vorgelegt werden, ist diese diskordant eingelagerte Deckfazies einwandfrei festzustellen, nur setzt sie eben nicht unmittelbar an äußeren Terrassenbord ein (so Rheinheim und Villigen).

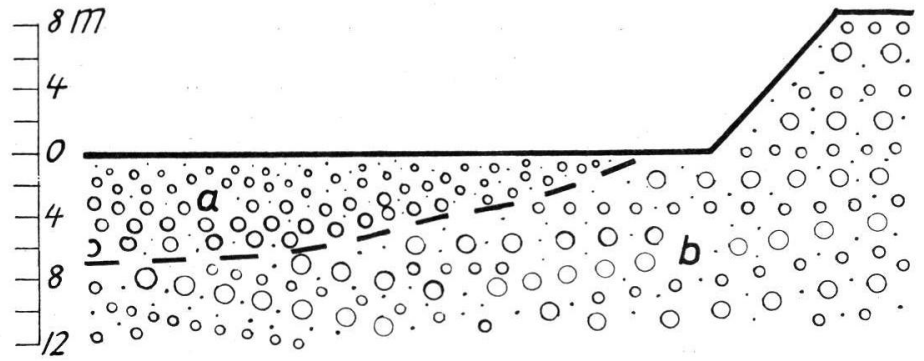

$\mathrm{Abb}$. 2. Schema der jüngeren Schottereinlagerung (a) in die letzteiszeitliche Hauptakkumulation im Hochrhein- und unteren Aaretal. - - - Diskordante Grenze zwischen älterem und jüngerem Schotter. - - Heutige Oberfläche der Niederterrassen-Treppe.

Handelt es sich dabei nun um Akkumulations- oder um Erosionsterrassen? Streiten wir des längeren darüber, ob die Decklage nur die bei Hochwasser bewegte TalbodenGerölldecke war, die vor neuerlichem Einschneiden des Flusses endgültig liegen blieb, daß sie also nur eine "An“-schüttung, aber keine "Auf“-schüttung darstelle (I. SCHAEfER)?

Folgende Tatsachen sprechen dafür, daß es sich bei allen diesen Flächen nicht um Erosionsformen handelt:

a) Die Einlagerung einer immerhin bis $8 \mathrm{~m}$ mächtig werdenden Gerölldecke.

b) Die Breite der Terrassen, ihr flaches Gefälle und die Möglichkeit, sie über weite Strecken, besonders unterhalb der Aaremündung, verbinden zu können.

c) Die Flächen zeigen keinerlei Unebenheiten, Altwasserbögen, Kolke, Rinnen oder dergleichen, sondern sie sind wahrhaft eben genau wie das oberste NT-Niveau, dem der Charakter einer Akkumulationsebene zugebilligt wird. Den Talböden, die auf Erosion beruhen, sieht man nämlich auch heute noch diese Genese an. Ihre unruhige Oberfläche wird oft von einem Hochwasser-Aumergel abgedeckt, der ihnen erst eine ebene Oberfläche zu geben vermag.

$\mathrm{Zu}$ 8. Schon 1912 hatte der Tiroler Geoluge O. Ampferen eine Korrektur der Auffassungen A. Penck's über die "Glaziale Serie“ vorgeschlagen. „Die Schotter sind durch die Flüsse, die Moränen durch die Gletscher geschaffen“" (aus I. Schatefer 1950, S. 52). Spätestens im Laufe der 30-er Jahre hatte sich eine ähnliche Auffassung in Deutschland durchgesetzt. Es ist vielleicht auf die Schweiz, vielleicht sogar nur auf die Nordschweiz zu be- 
schränken, wenn Mosen meint, die Vorstellung von der Glazialen Serie müsse revidiert werden (S. 93). Allerdings ist sein abgebildetes Blockdiagramm das richtigste über diesen Gegenstand.

$\mathrm{Zu}$ 9. Die von Moser zitierte Vorstellung gilt seit je nicht für die äußersten und flachsten Teilzungenbecken, da zu einer Drumlinisierung des Untergrundes eine gewisse Gletschermächtigkeit nötig ist (mindestens $4-600 \mathrm{~m}$ ). Es ist auch seit längerem bekannt, daß nur die Ränder eines Teilzungenbeckens oder die Schwellen zwischen zwei hintereinanderliegenden Becken drumlinisiert werden, während die zentralen Teile der Becken der Ausschürfung durch das Eis unterlagen. In den schmalen, übertieften Talbecken des Schweizer Mittellandes ist von vornherein die Möglichkeit einer Drumlinisierung stark eingeschränkt, an den Gletscherenden am Innensaum der Endmoränen aber garnicht zu erwarten.

$\mathrm{Zu}$ 13. Über die Gliederung der Rißeiszeit ist ja noch lange nicht das letzte Wort gesprochen. Wir wissen, daß ostwärts des Rheingletschers die Frage der Altersstellung von Riß I (Weidenbach = etwa Altriß von Graul) zu Mindel II = maximale Vergletscherung in Bayern (Eberl, Knauer) noch nicht gelöst ist. Sollte sich in der von Graul, Schaefer \& Weidenbach (1951) vermuteten Weise eine Altersgleichheit dieser heute noch verschieden benannten Moränenablagerungen bewahrheiten, dann wäre ein echtes Interglazial zwischen Alt- und Mittelriß (R I/R II der Schweiz) anzunehmen. Daneben gibt es aber aus dem Gebiet westlich des Rißtales eine ganze Reihe von geologischen Beweisen für eine echte Warmzeit vor der Riß II = Schweizer Maximalvereisung.

$\mathrm{Zu}$ 14. Obwohl schon A. Penck und E. Brückner auf die Tatsache der glazialen Übertiefung auch im Schweizer Mittelland hingewiesen haben, ist $\mathrm{m}$. E. dieser Faktor für die Erklärung der hier gegenüber dem bairisch-österreichischen Alpenvorland in der Regel anderen Höhenlage-Abfolge der fluvioglazialen Schottersohlen zu wenig berücksichtigt worden. Die von A. Heim geradezu fanatisch verfochtene Antithese von einer geringfügigen Glazialerosion führte ihn bekanntlich zu ziemlich gekünstelten Vorstellungen tektonischer Vorgänge am Alpenrand und im Alpenvorland, die in ihren krassesten Fällen inzwischen durch die Schweizer Molasseforschung richtiggestellt wurden. Aber auch die Thesen von den "Rinnenschottern“, die vielfach, wenigstens in ihren unteren Abteilungen, als tektonische und nicht glaziale Ablagerungen aufgefaßt werden, wie die der Mittelterrassenschotter (siehe dazu noch R. HubER's Schematische Erosions- und Akkumulationskurve S. 81) gehen wenigstens z. T. noch auf jene A. Heim'sche Antithese zurück. Wenn wir aber berücksichtigen, daß das gesamte Schweizer Mittelland zumindest einmal, wenn nicht mehrmals total von Eis erfüllt war, und für die größeren Täler nur einen mittelpleistozänen Eisausschurf von wenigen Zehnern von Metern annehmen, dann ist die tiefe Lage und große Mächtigkeit der nachträglichen Auffüllungen jener übertieften Talstrecken auch ohne ein tektonisches Auf und $\mathrm{Ab}$ des Mittellandes zu verstehen. Nötig ist dabei natürlich der Nachweis einer ziemlich weit ins Vorland herabreichenden Vergletscherung vor der Maximalvereisung. Wir besitzen sie in unserem Altriß, das durch sehr mächtige Ablagerungen repräsentiert wird. Ich meine also, die nur auf tektonischen Vor-gängen beruhende Erklärung der tiefen Lage mittelpleistozäner Mittelland-Schotter scheint noch revisionsfähig zu sein. Die extramoränen "Mittelterrassenschotter" (A. WEBER, H. Suter, R. Huber, H. Jäckli u. a.) sind bereits als Würm-Vorstoßschotter erkannt worden. Wo liegen die R II-Vorstoßschotter, die z. B. bei Biberach/Riß 40-50 m mächtig sind?

Schluß or t: Dieser Diskussionsbeitrag könnte noch in einzelnen Punkten fortgesetzt werden, so vor allem über die Definition der „abgleitenden Erosionsflächen“, über die Frage der Seitenerosion bei Akkumulation, über die Erklärung der Grobschotterlagen innerhalb der fluvioglazialen Akkumulationen und mehr. Doch würde dies unweigerlich

7 Eiszeit und Gegenwart 
in zu lange und zu spezielle Überlegungen führen, die hier keineswegs beabsichtigt waren. Vielmehr wollte ich, wie schon eingangs gesagt wurde, auf die prächtigen Arbeiten junger Schweizer Geomorphologen hinweisen und die Hoffnung ausdrücken, daß jene dicht auf der Spur bleiben mögen, die sie so sicher zu verfolgen begonnen haben.

L i te r a t u r (nur die mit Erscheinungsjahr zitierte):

BECK, H.: Glazialmorphologische Untersuchungen in der Gegend von Solothurn. - Diss. Bern 1957. BöGLI, H.: Die Herbstexkursion 1954 (der Schweizer Geomorph. Ges.). - Geogr. Helv. 10, Zürich 1955.

Boesch, H.: Bemerkungen zum Terrassenbegriff. - Tijdschr. kon. nederl. Aardrijksk. Gen. 74, 1957.

Bübel, J.: Die morphologischen Wirkungen des Eiszeitklimas im gletscherfreien Gebiet. - Geol. Rdsch. 34, S. 482 ff., Stuttgart 1944.

ERB, L.: Zur Stratigraphie des mittleren und jüngeren Diluviums in Südwestdeutschland und dem schweizerischen Grenzgebiet. - Mitt. bad. geol. L. A. Freiburg i. Br. 11, 1936.

Graul, H.: Uber die quartären Geröllfazien im deutschen Alpenvorlande. - Geolog. Bavar. 19, S. 266, München 1953.

Graul, H.; Schaefer, I. \& Weidenbach, F.: Quartärgeologische Exkursion durch die Riß-Lechplatte. - Geolog. Bavar. 6, S. 97, 1951.

HantKe, R.: Zur Phasenfolge der Hochwürmeiszeit des Linth- und des Reuss-Systems, verglichen mit derjenigen des Inn- und des Salzach-Systems sowie mit der nordeuropäischen Vereisung. - Vierteljschr. naturf. Ges. Zürich 104, S. 390, 1959. - - Zur Altersfrage der Mittelterrassenschotter. Die riß-würm-interglazialen Bildungen im Linth/Rhein-System und ihre Äquivalente im Aare/Rhone-System. - Vierteljschr. naturf. Ges. Zürich 104, 1959.

HUBER, R.: Ablagerungen aus der Würmeiszeit im Rheintal zwischen Bodensee und Aare. - Vierteljschr. naturf. Ges. Zürich 101, S. 1, 1956.

Hug, J.: Die Zweiteilung der Niederterrasse im Rheintal zwischen Schaffhausen und Basel. Zt. f. Gletscherk. 3, 1909.

JäckLI, H.: Wurde das Moränenstadium von Schlieren überfahren? - Geogr. Helv. 14, Zürich 1959.

KimbaLI, D., \& ZeUner, F. E.: The terraces of the Upper Rhine and the age of the Magdalenian. Univ. of London, Inst. of Arch. 1946.

KnAUER, J.: OUber die zeitliche Einordnung der Moränen der „Zürich-Phase“ im Reussgletschergebiet. - Geogr. Helv. 9, 1954.

Schaefer, I.: Die diluviale Erosion und Akkumulation. - Forsch. z. dt. Landesk. 49, Stuttgart 1950 SoERgeL, W.: Die Ursachen der diluvialen Aufschüttung und Erosion. - Berlin 1920.

Trols, C.: Die jungglazialen Schotterfluren im Umkreis der deutschen Alpen. - Forsch. z. dt. Landes- u. Volksk. 24, Stuttgart 1926.

Manuskr. eingeg. 14. 5. 1960.

Anschrift des Verf.: Professor Dr. Hans Graul, Heidelberg, Dantestraße 8. 